\title{
Rare Wild Animal Tracking in the Forest area with Wireless Sensor Network in Network Simulator-2
}

\author{
Sanjyot S. Ghumare \\ ME in Microwave \\ Amrutvahini College of \\ Engineering, Sangamner, \\ Ahmadnagar, India
}

\author{
Rekha P. Labade \\ HOD of E\&TC \\ Amrutvahini College of \\ Engineering, Sangamner, \\ Ahmadnagar, India
}

\author{
Sunil R. Gagare \\ Asst. Prof of E\& TC \\ Amrutvahini College of \\ Engineering, Sangamner, \\ Ahmadnagar, India
}

\begin{abstract}
To keep of track wild animal in forest area is very difficult task. As one cannot go to the deep inside forest, as no one wants to jeopardize one's life. Use of Wireless sensors in such areas is best solution. As these sensors only have to put in one place and after that they self-organize their position in the given or programmed area. To track an animal Localization is very important factor. As if the position of the sensor nodes are known in the sensor network, it is easy to identify position of an animal in that network. In wireless sensor network energy saving is also an important factor for networks lifetime. Here clustering algorithm is using to save energy in the nodes which is Low Energy Adaptive Clustering Hierarchy along with prediction operation in the area. Spatial resolution is also measure in this simulation for checking accuracy in the animal tracking.
\end{abstract}

\section{General Terms}

LEACH, Spatial Resolution.

\section{Keywords}

Animals Trajectory finder, Prediction, clustering, NS-2.

\section{INTRODUCTION}

Wireless sensor network becoming a popular technology this days. WSN has many applications in different areas like infrastructure security, habitat monitoring, Indoor systems and traffic control [1]. Target tracking with wireless sensor network over large area is reliable option now a days. In critical places use of wireless sensor network is the best option. Cost is not issue in WSN, is a cost saving technique comparatively with other communication techniques. Here in this paper the results are showed on animal tracking with wireless and mobile sensor network. Real time and fast results is requirement for today's world and this is getting here in this work. Localization means to know the position of the sensors node in the WSN, which is very necessary to be known for tracking [2]. As two techniques are in localization from which Range free localization concept is used in this paper instead of range based localization, because of its required advantages in this scenario. Range free localization is useful in this scenario because of its working without GPS in the system. As in forest area there is probably one can't use GPS. With the use location based routing technique which is an option where one can't use GPS, here considered sensor node location as their address. For reliable operation in the network like scalability and energy saving Clustering approach that is Low energy adaptive clustering hierarchy (LEACH) is an algorithm for clustering is used. To use minimum nodes for tracking the target prediction operation is used here [3]. In this work
Network simulator-2 (NS-2) software is used, for simulation purpose consideration for mobility in the animal's behavior in the area of WSN is necessary. Individual mobility pattern is considered in this paper. Mobility model which can show the animals behavior is random way point mobility model. Random waypoint mobility model is popular in the group of individual mobility model and is randomly select its destination and starting point in the network [4].

This problem is similar to the target tracking and many did work in this area. Data loss is important factor considered while Target tracking with localization [4]. For tracking mobile target, More than one mobile robots are in the scenario which are mobile and going to track by the other robots which are stationary [5]. In [3] target tracking is done by giving preference to the seamless as well as fast handover with using zig-bee (802.15.4) and concentrating on media access control layer. To track the target, as the mobile target plan is unknown, time of arrival (TOA) measurements from the mobile sensor network is used by the mobile sensor controller. Mobile sensor controller collect TOA is obtained from both the mobile target and mobile sensor to direct mobile sensor to follow the target and also to estimate location. To estimate the location min max approach is used [3] [6]. For localization purpose GPS is used in the sensor network nodes while tracking target accurately [7].

\section{PROPOSED WORK}

This work of target tracking, range free localization is used, which is of low cost and minimum hardware requirement. LEACH algorithm with prediction technique is used for the long life of the network. For mobility of the target random waypoint model is used. To calculate distance between two nodes with respect to the incoming signal strength to predict the path. For organization of the nodes in the WSN signal strength is important factor.

\section{SIMULATION MODEL}

In this paper of Target tracking, NS2 software is using here for simulation. In that software, initially deploy sensors at a fix position and then network auto-adjust the nodes, here adjusting nodes in Grid format on Rectangular area with 2diamentional format as shown in the fig.1. Dividing nodes in groups called as a cluster. Node- 1 to Node 9 are forms a cluster like-wise node 10-18, 19-27, 28-37 these groups form clusters in network. Cluster head is selected by sensor nodes in the cluster. Each cluster head know the position of every sensor in the cluster. If the cluster is continuously working as the animal which is tracked in present, in the surveillance, then cluster head is elected time to time for improvement of network life time. 


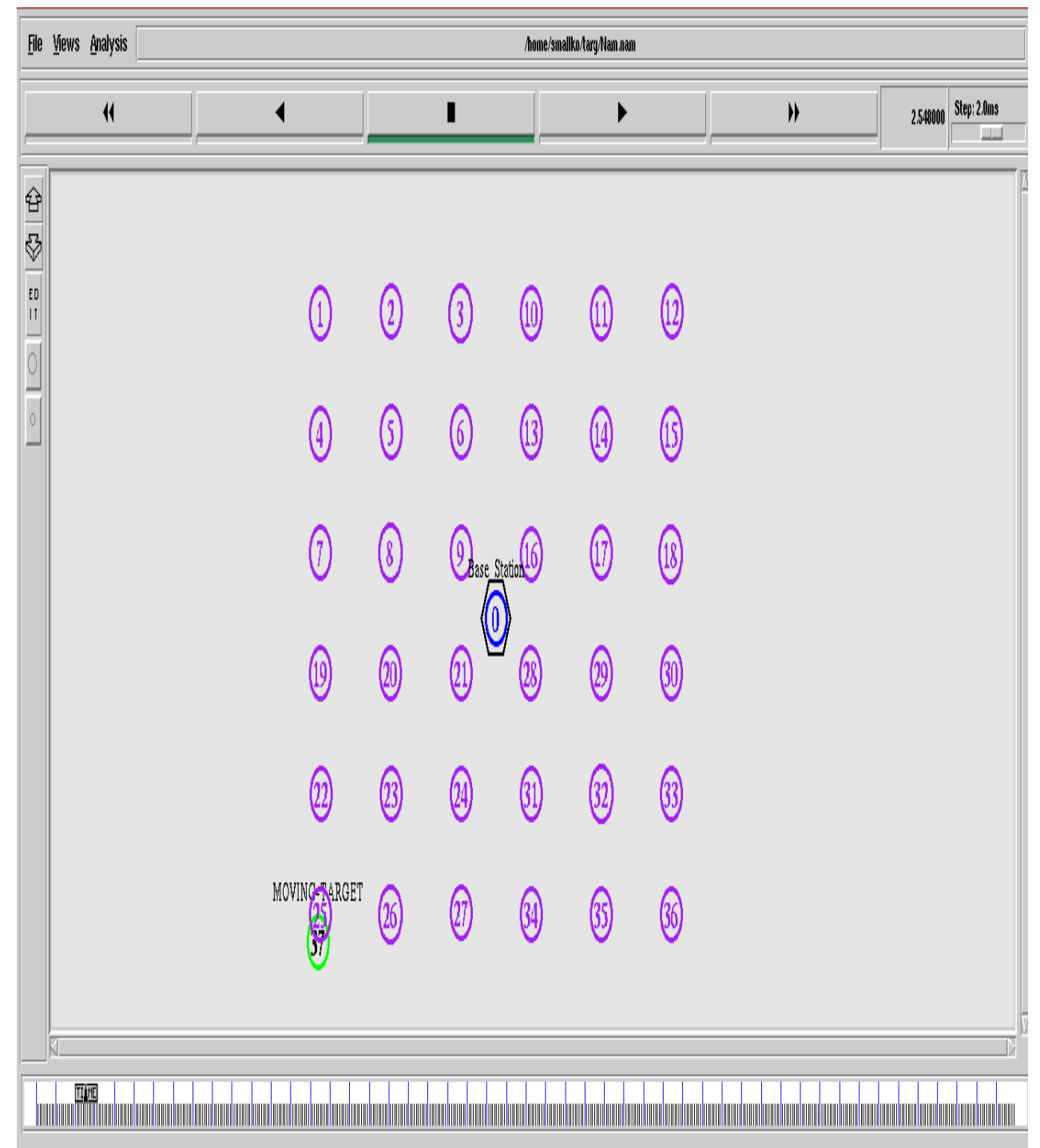

Figure 1: Deployment of the nodes in WSN

For initial calculation of the distance between two nodes, Pythagorean Theorem is used as shown below

$$
d=\sqrt{\left(x_{2}-x_{1}\right)^{2}+\left(y_{2}-y_{1}\right)^{2}}
$$

Where, $\mathrm{d}$ is a distance between two nodes and the position of one node is $\left(\mathrm{x}_{1}, \mathrm{y}_{1}\right)$ and other node is $\left(\mathrm{x}_{2} \mathrm{y}_{2}\right)$. Calculation Then Adding Mobility to the Target Node, where target node is mobile in random direction from 0 to $2 \pi$ also with random speed from 0 to $v_{\min }^{\max }$ where $\mathrm{v}$, is velocity. Then Make clusters with all nodes in the network by using LEACH algorithm. When Target is Track by a sensor in a cluster where target is present Node forward that data to cluster head. Cluster Head then pass that data to the base station. Also information is predicted about a target to nearby cluster.

Showing the results for following different parameters in NS2 software:

- $\quad$ Area size (A) - $1200 * 900 \mathrm{~m}^{2}$;

- $\quad$ Number of Mobile Sensors (N(A))-36;
- $\quad$ Number of Target - 1 ;

- $\quad$ Target Nodes Speed -30ms

- $\quad$ Each Sensor Nodes power- $0.05 \mathrm{db}$

- Total Power of the Network- $0.35 \mathrm{db}$

- $\quad$ Each Sensor Nodes coverage range- $281 \mathrm{~m}$

- Mobility Pattern of Target - Random waypoint mobility.

\section{RESULT AND PERFORMANCE ANALYSIS}

Figure 2 shows that how this network process in the given amount of time that is throughput of the WSN. Spatial resolution is shown in the figure 3 as Targets travelling distance without detected by sensors. As Spatial resolution is defined as the sensors even when in the area of surveillance but not detected by any sensor node. 


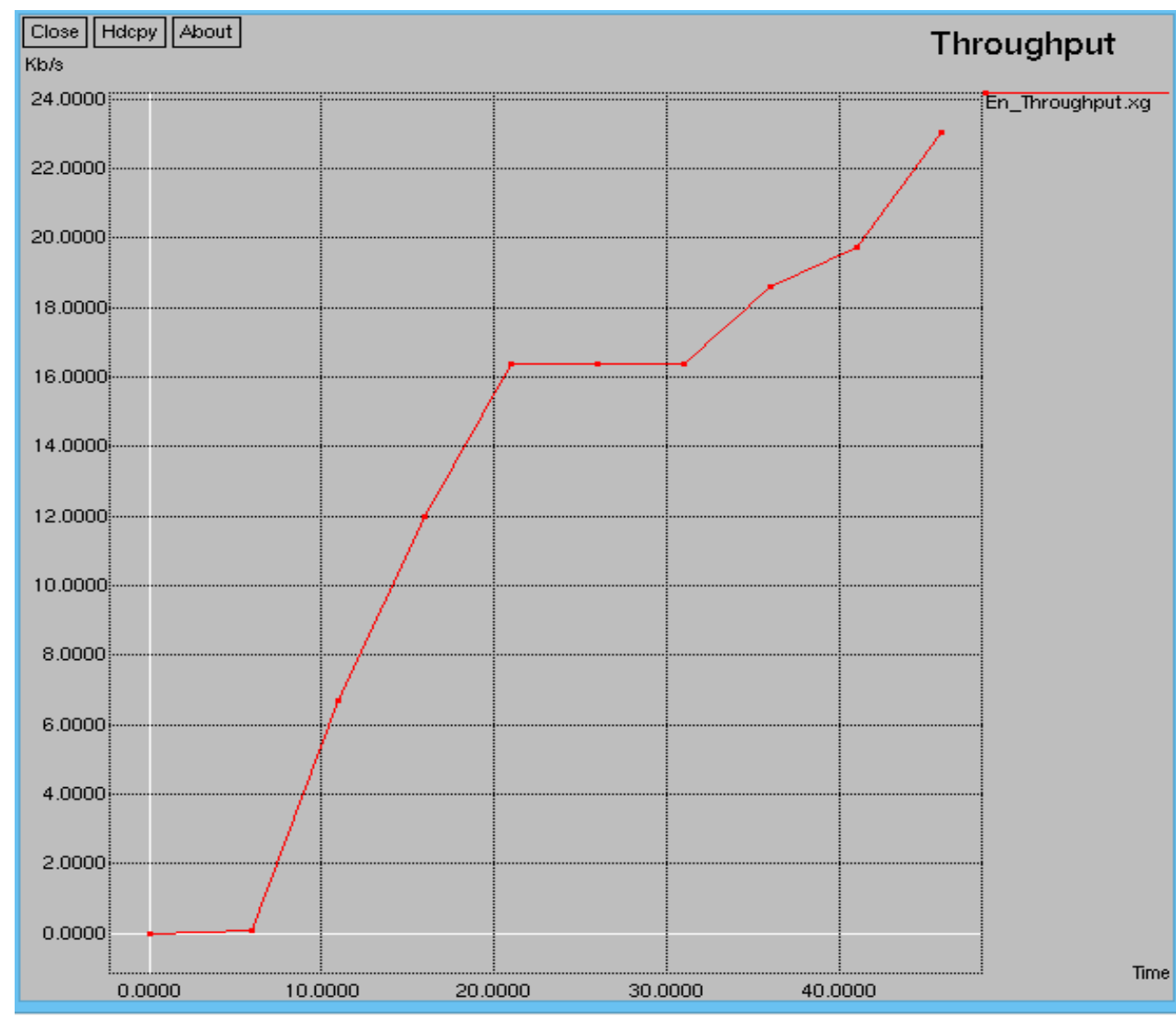

Figure 2: ThroughPut

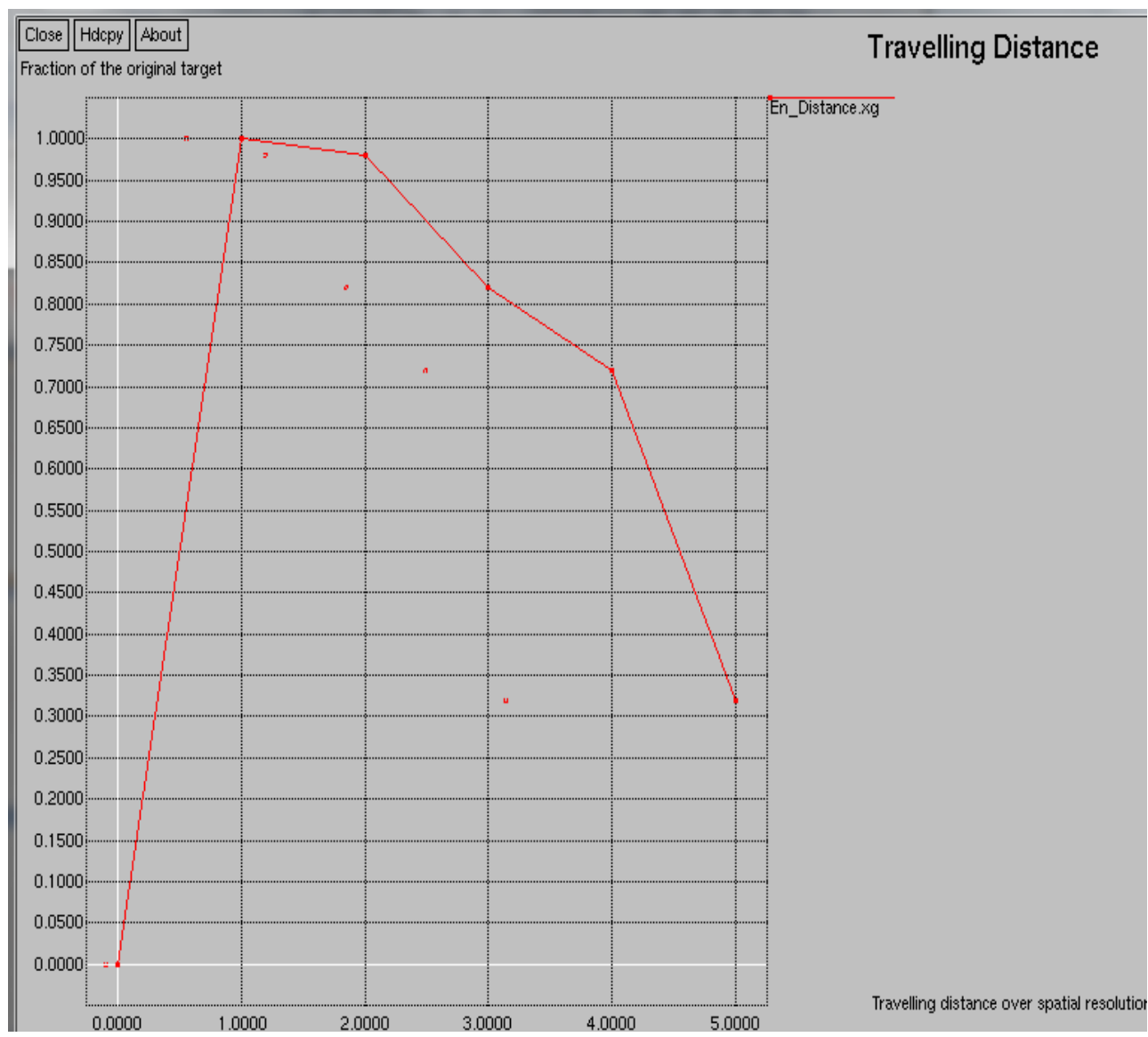

Figure 3: Targets travelling distance without detected by sensors 


\section{CONCLUSION}

Moving animal is get successfully track in this simulation with NS-2 software. Good throughput from this work is getting without a use of satellite or globalization of node. Implemented LEACH algorithm successfully for energy saving in the network. Prediction is also done in this work to improve networks life span. Spatial resolution is calculated for accuracy of finding animal's trajectory in the surveillance area. One can add mobility to sensors to improve the networks life-time. Also it will reduce number of nodes.

\section{REFERENCES}

[1] Chi-ChangChen, Chi-YuChang and Yan-NongLi, "Range-Free Localization Scheme in Wireless Sensor Networks Based on Bilateration," p. 10, 2013.

[2] Himakshi and C. K. Singh, "A Review: Wireless Sensor Networks Application and Technology," International Journal of Computing \& Business Research, 2012.

[3] E. Xu, Z. Ding and S. Dasgupta, "Target Tracking and Mobile Sensor Navigation in Wireless Sensor Networks," in IEEE transactions on mobile computing, 2013.

[4] G. Raghunandan and B. Lakshmi, "A Comparative Analysis of Routing Techniques for Wireless Sensor
Network," in Proceedings of the National Conference on Innovations in Emerging Technology, 2011.

[5] Sikora and E. Niewiadomska-Szynkiewic, "Mobility Model for Self-Configuring Mobile Sensor Network," in International Conference on Sensor Technologies and Applications, 2011.

[6] V. P. Sadaphal and B. N. Jain, "Tracking mobile target using selected sensors," 2014

[7] E. T. Yazdi, A. Moravejosharieh and S. K. Ray, "Study of Target Tracking and Handover in Mobile Wireless Sensor Network," in IEEE ICOIN, 2014.

[8] J. Suh, S. You and a. S. Oh, "A Cooperative Localization Algorithm for Mobile Sensor Networks," in 8th IEEE International Conference on Automation Science and Engineering, Seoul, Korea, 2012.

[9] B. Zack and R. Daniela, "Event-Based Motion Control for Mobile- Sensor Networks," in Published by the IEEE CS and IEEE ComSoc, 2003.

[10] G. Y. Keung, B. Li, Q. Zhang and H.-D. Yang, "The Target Tracking in Mobile Sensor Networks," Networking, 